\title{
Low-amplitude dynamic tests of fine gravel in a resonant column
}

\author{
Zaven Ter-Martirosyan, Evgeny Sobolev" and George Anzhelo \\ Moscow State University of Civil Engineering, Yaroslavskoe shosse, 26, Moscow, 129337, Russia
}

\begin{abstract}
Construction of industrial and civil buildings, taking into account the dynamic effects on the foundations, requires special experiments on the mechanical properties of soils. This article presents the results of studying the dynamic properties of coarse gravelly soils using the resonant column method. These studies are relevant, since the determination of the dynamic properties of coarse-grained soils under laboratory conditions is associated with a restriction on the size of the fractions in the sample volume. This circumstance leads to the fact that at the moment most of the laboratory tests of the dynamic properties of coarse-grained soils are performed on smaller aggregate fractions, which, in general, significantly reduces the resulting mechanical properties of soils. It does not reflect the real operation of the foundation of buildings during dynamic effects. This paper presents a description of the available laboratory equipment, the sequence of preparation of samples of coarse grained crushed stone soil and sample assembly in the working chamber of the installation. The article contains the main graphs characterizing the change in shear modulus and damping coefficient depending on shear deformations. It is noted that the results obtained are particularly relevant for modeling the dynamic effects of natural and man-made character on the foundations of industrial and civil buildings, the bases of which are composed of coarse-grained soils. Dynamic parameters considered in this paper, can and must be used in numerical calculations by finite element method with the use of modern groundwater models in geotechnical software systems.
\end{abstract}

\section{Introduction}

Engineering surveys and design of the foundations of industrial and civil buildings in seismic areas require special types of studies of the dynamic properties of soils. And without that, not an easy task becomes more complicated when designing bases composed of coarse-grained soils lying within the limits of the compressible strata. Such bases can be represented by gravel (rounded) or crushed stone (angular) coarse-grained particles of rocks [1-3].

Currently, considerable experience has been gained in the study of the dynamic properties of sandy and clayey soils both in laboratory and in field conditions. However, the

\footnotetext{
* Corresponding author: e.s.sobolev@mail.ru
} 
results of the determination of stiffness and damping for coarse soils are presented not so much. The main problem of studying the dynamic properties of coarse soils in the laboratory is the limitation on the size of the inclusions allowed in the soil sample. The diameter of the sample for research is chosen so that the maximum size of the inclusions does not exceed $1 / 6$ of its diameter [4-7]. In practice, studies of the mechanical properties of coarse-grained soils are often carried out completely and completely by «aggregate», thus obviously worsening the parameters obtained during the tests $[8,9]$.

Focus on laboratory studies, among which the most common should be noted tests of the method of dynamic triaxial compression and resonant columns. The method of dynamic triaxial compression is less suitable for determining the dynamic properties of coarsegrained soils, since there are not always realistic requirements on the accuracy of displacement measurements. It is necessary to use LVDT-strain sensors on a local base, allowing to ensure the specified measurement accuracy. Such tests are very time consuming.

Laboratory studies of coarse-grained soils by the method of low-amplitude dynamic tests in a resonant column are also busy, however, they allow the analysis of dynamic properties with high accuracy. The range of measurement of shear deformations in resonant columns is much wider than in the conditions of dynamic triaxial compression. Studies in resonant columns are carried out to determine: the speed of transverse waves $(\mathrm{Vs}, \mathrm{m} / \mathrm{s})$, the dynamic shear modulus (G, MPa) and the damping coefficient $(\mathrm{D}, \%)$ of the soil in the range of shear strains of the order of $10^{-4}-10^{-2} \%$, as well as to study the changes in these indicators in the range of small shear deformations (not more than $0,1 \%$ ) in order to calculate the vibrations of structures under dynamic effects [10-12].

\section{Methods}

Prepared samples of limestone rubble in the form of a continuous cylinder were placed in a chamber of three axial compression and, after preliminary isotropic consolidation, were subjected to dynamic loading by applying torsional vibrations of variable frequency to the upper - free end of the sample. In the process of oscillation, the soil undergoes a simple shear deformation. The amplitude of the shear deformation is determined at each frequency of the specified range, which allows to obtain the amplitude spectrum of the soil reaction and determine the resonant frequency of the sample. By first natural frequency of the sample is calculated shear wave velocity in it $\left(V_{s},\right)$, and then a dynamic shear modulus $(\mathrm{G})$.

The soil damping coefficient (D) is determined by the damping of free oscillations, or by the width of the resonance curve near the resonance with forced oscillations, and the absorption is assumed to be classical in nature. During the experiment, the amplitude of the dynamic load gradually increases, which allows determining the changes in the shear modulus (decrease) and the absorption coefficient (increase) with an increase in the achieved amplitude of shear deformations $[13,14]$.

Dynamic tests of coarse-grained soils by the method of a resonant column were carried out according to a consolidated undrained scheme, since any change in the shape or mass of the sample during the test changes its inertia moment and distorts the final result $[15,16]$.

The study used a resonant column with an electromagnetic system for creating torsional vibrations, the drive of which is located inside the chamber of three-axis compression. In addition, there are devices for measuring small angular movements (turns) of the sample, which are accelerometers. The loading system provides the excitation of torsional vibrations in the range from 0 to $200 \mathrm{~Hz}$. General view of the installation is shown in Figure 1. 

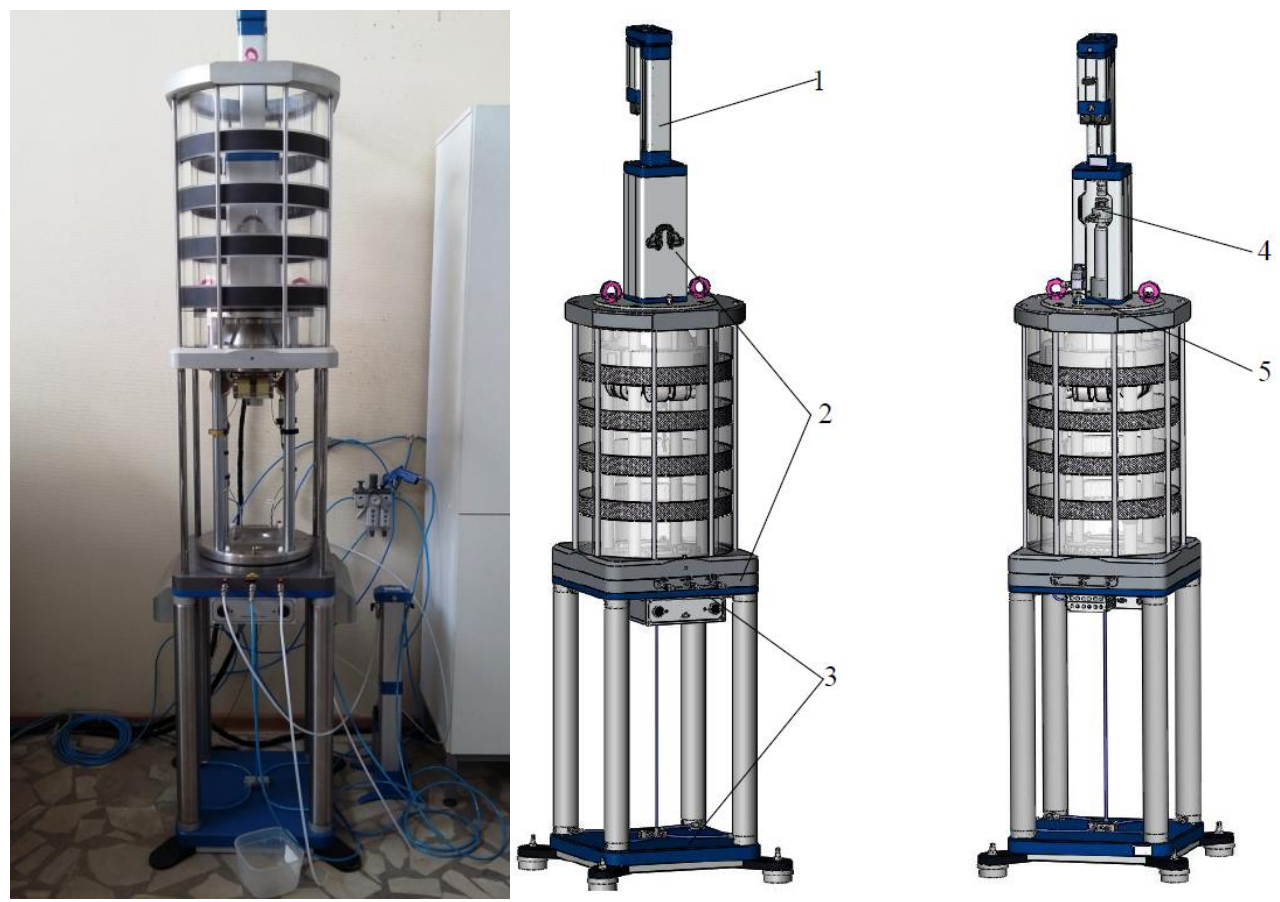

Fig. 1. General view of the resonant column available at MSUCE (Moscow, Russia). The numbers indicate: 1 - the actuator; 2 - the camera; 3 - lifting device; 4 - force sensor; 5 - pressure sensor

Inside the three-axis compression chamber there is a soil sample, an active plate with a top die, an accelerometer, magnetoelectric drives, a displacement sensor. Four magnetoelectric drives and a vibration transducer are installed on the upper active plate, creating a torque (Figure 2).

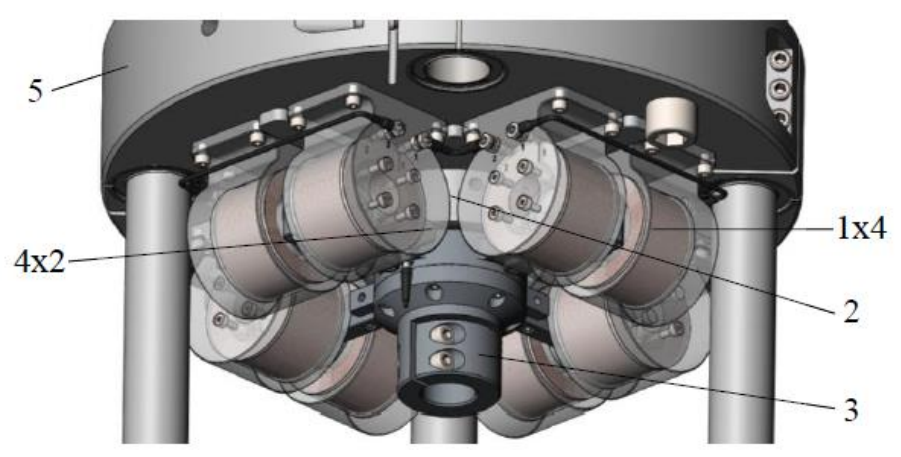

Fig. 2. Diagram of the upper active plate of the resonant column. Numbers indicate: 1 magnetoelectric drive; 2 - bracket; 3 - clamp; 4 - vibration transducers; 5 - plate

Formation of samples using the method of dry layered dumping was carried out in a cylindrical detachable form-template (Figure 3), installed directly on the pedestal of the camera of the three-axis compression device, with a latex membrane stretched on its inner surface. Since the granules of limestone rubble have sharp edges, to avoid damage to the latex membrane, smaller particles are poured between larger particles, and then sandy aggregate is poured between smaller particles. When tapping with a rubber hammer on the 
base of the chamber, a tighter soil build was achieved. After installing the stamp and fixing the upper end of the membrane on it, but before filling the chamber of the device with water, a vacuum is applied to the sample to increase the effective stresses and preserve their homogeneity through the upper drainage line. The lower drainage line remains blocked. After that, the upper drainage line was hermetically sealed, the detachable form-template was removed [17-19].

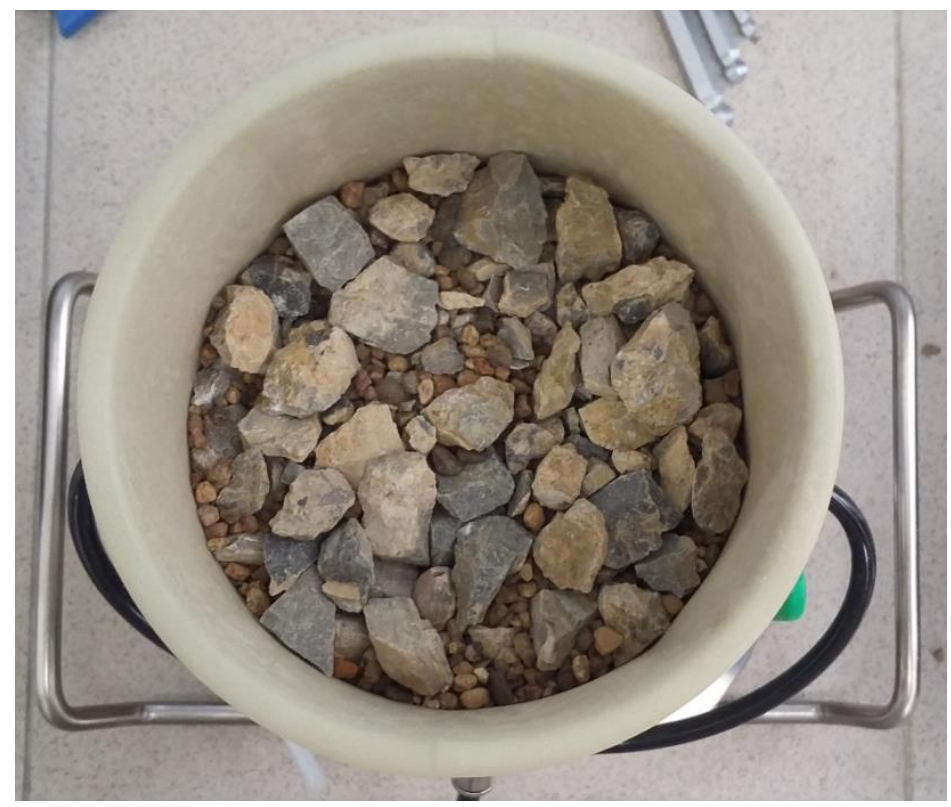

Fig. 3.Template form with a latex membrane at the time of filling the soil sample

Water saturation of initially dry coarse-grained soil was carried out directly in the device chamber. First, the sample was subjected to hydrostatic compression to $\sigma_{3}=10 \ldots 15$ $\mathrm{kPa}$ with simultaneous removal of vacuum in order to avoid radial deformations. The sample was filled with distilled and pre-evacuated water for complete degassing with water from below with a pressure of about $30 \mathrm{~cm}$ until the evolution of gas bubbles with water through the upper drain line ceased. Such preparation allows to obtain fully water-saturated samples with the Skempton parameter $\beta=0.94 \ldots 1.00$ (depending on their density of addition).

After the consolidation was completed, the drainage was blocked and the soil sample was tested with the specified dynamic load parameters that are not associated with the expected dynamic effects on the soil foundation and are created to determine the characteristics of the soil as an environment of propagation of oscillations. The amplitude of dynamic stresses is given by the magnitude of the torque applied to the upper (free) end of the sample. The range of oscillation frequencies in which resonance is expected, and the frequency change step during the experiment was set by selection, based on the expected response of the soil and is adjusted in subsequent tests. During the experiment, we measured the maximum values of the angular displacements (turns) at each step of changing the oscillation frequency [20].

The initial torque was chosen in such a way that the resulting shear deformations were as small as possible, but at the same time allowed to fix the resonant frequency with confidence. It should be noted that the magnitude of the initial torque also depends on the magnitude of the compressive stresses. The frequency range was taken from 20 to $200 \mathrm{~Hz}$. 
At each frequency of the selected range, 5-10 cycles of oscillations were carried out to obtain a stable reaction of the soil [21].

After the completion of the forced oscillations at each step of loading, the damping of the free oscillations of the sample was recorded, the change in the amplitude of which allows the damping coefficient to be calculated later.

\section{Results}

Data processing consisted in calculating for all steps of dynamic loading the relative shear deformations $\gamma$, determining its value $\tau \max$ at the resonant frequency $\omega$ and calculating for this frequency the velocity of the transverse waves $\mathrm{V}_{\mathrm{s}}$ and then determining the dynamic shear modulus $\mathrm{G}$ and the absorption coefficient $\mathrm{D}$.

The relative shear strain $\gamma$ is calculated from the measured angular displacements as the skew angle tangent $\Theta$ at a point remote from the center of the sample at $0,78 \mathrm{r}_{0}$ for solid samples at each step of changing the excitation frequency. According to the results of the calculation, a resonance curve is constructed, from which the value of the resonant oscillation frequency $\omega$ is taken at $\gamma=\gamma_{\max }$.

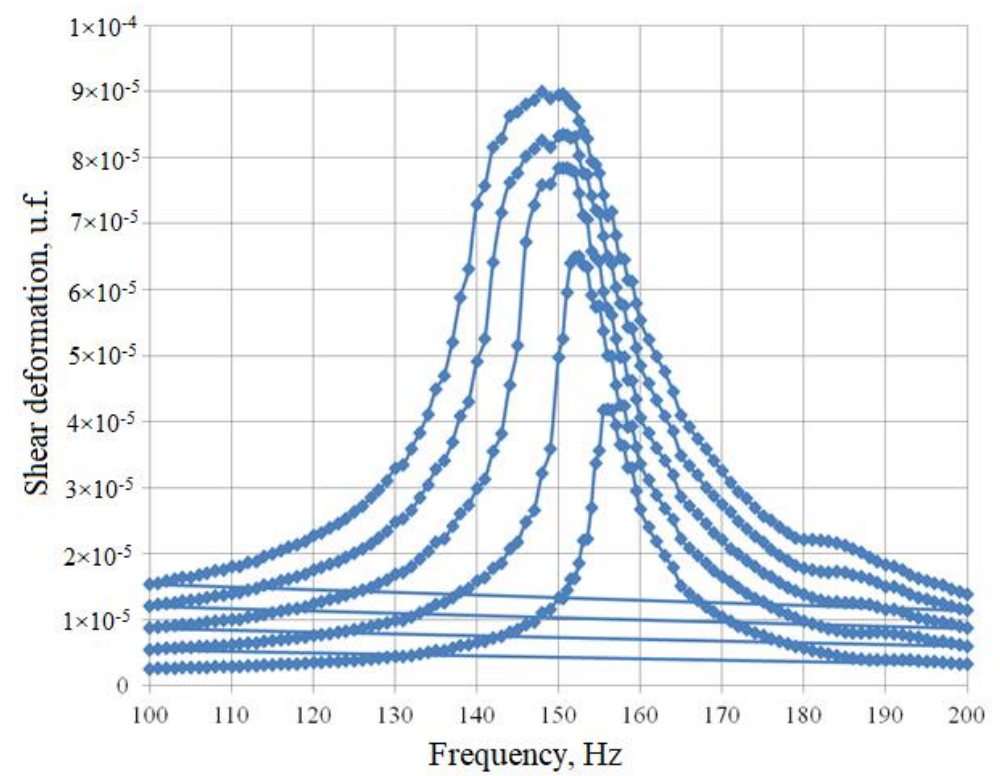

Fig. 4. Graph of shear strain $\gamma$ (u.f.) versus frequency $\omega(\mathrm{Hz})$ used to calculate shear modulus $\mathrm{G}(\mathrm{kPa})$

The value of the velocity of the transverse waves $\mathrm{V}_{\mathrm{s}}$ is calculated from

$$
V_{s}=\omega h\left(\frac{J}{J_{0}}\right)^{\frac{1}{2}}
$$

where - $\mathbf{J}$ is the moment of inertia of the sample (calculated from the mass and geometric dimensions of the sample in accordance with the known physical relationships at the time of the end of consolidation) for a solid cylindrical sample of mass $\mathrm{m}$ and radius $\mathrm{r}$ $\mathrm{J}=0,5 \mathrm{mr}^{2} ; \mathrm{J}_{0}$ - the moment of inertia of the power drive of the installation (indicated by the manufacturer in the documentation of the device), $\mathrm{h}$ - sample height, $\omega$ - resonance frequency. 
Having determined from (1) the velocity of the transverse wave $\mathrm{V}_{\mathrm{s}}$, the dynamic shear modulus is calculated from

$$
G=\rho V_{s}^{2}
$$

where $\rho$ is the density of the soil.

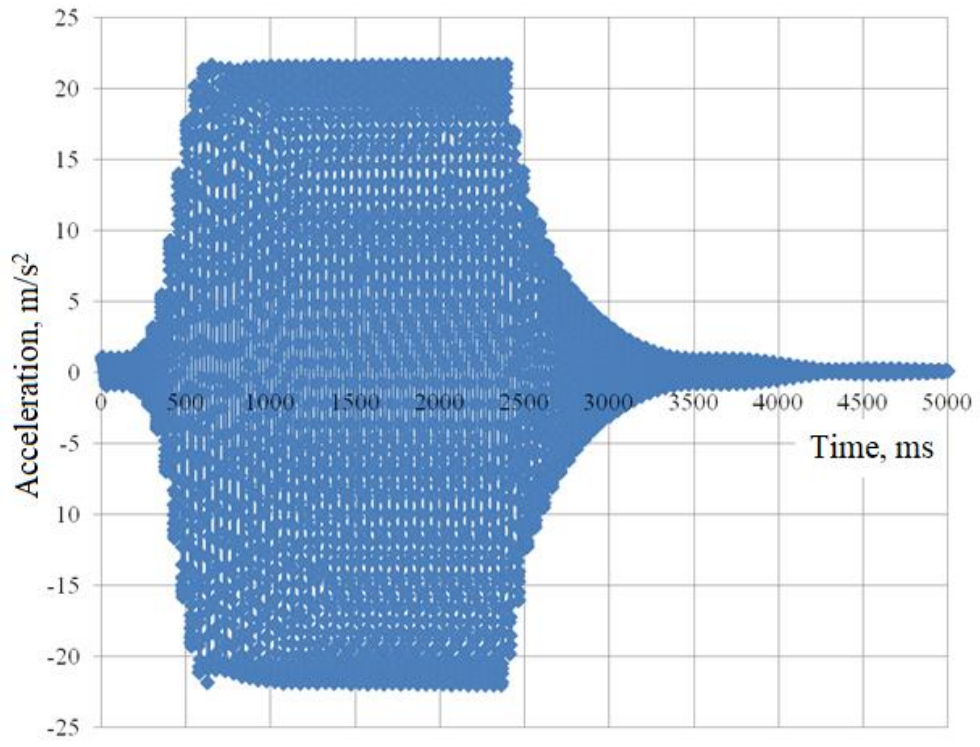

Fig. 5. Graph of acceleration a $\left(\mathrm{m} / \mathrm{s}^{2}\right)$ versus time $\mathrm{t}(\mathrm{ms})$, used to calculate the damping coefficient $\mathrm{D}(\%)$

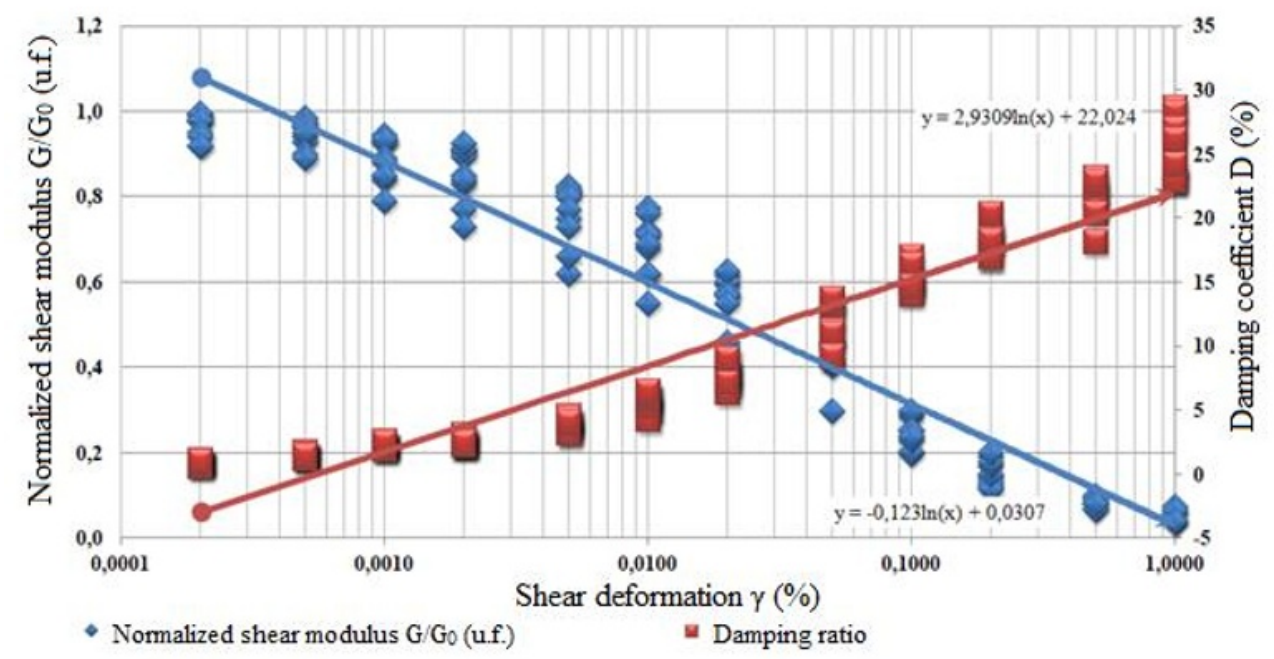

Fig. 6 .Graphs of the dependence of the normalized shear modulus $G / G_{0}$ (u.f.) and the damping coefficient $\mathrm{D}(\%)$ on the shear strain $\gamma(\%)$ for coarse-grained soil

According to the results of observations of the damping of free oscillations in time, a graph of the variation in time of shear deformations is built (Figure 5), based on the peak values of which a linear dependence is constructed $\gamma_{\max }(\mathrm{t})=\gamma_{0^{-}} \delta \square \ln (\mathrm{t})$, where $\delta$ is the logarithmic damping decrement, $\gamma_{0}$ is the peak value of the shear strain in the 1 st cycle. The 
absorption coefficient (D), estimated as a percentage of the critical attenuation and used, in particular, to take into account the damping properties of the base is calculated from

$$
D=\sqrt{\frac{\delta^{2}}{4 \pi^{2}+\delta^{2}}} .
$$

Further analysis is reduced to the construction and study of the dependences of the dynamic shear modulus and the absorption coefficient on the level of the resulting shear deformations (Figure 6).

\section{Conclusions}

Based on the results of special laboratory studies performed on the dynamic properties of coarse soils, the following main conclusions can be made.

1. The difficulty of studying the dynamic properties of coarse soils in the laboratory is the restriction on the size of large fractions in the sample volume. In this regard, tests are carried out on the placeholder, which ultimately leads to a deterioration in the resulting parameters.

2. Tests using the method of resonant columns allow to determine the speed of transverse waves $\left(\mathrm{V}_{\mathrm{S}}, \mathrm{m} / \mathrm{s}\right)$, the dynamic shear modulus $(\mathrm{G}, \mathrm{MPa})$ and the absorption coefficient (damping) $(\mathrm{D}, \%)$ of the soil in the range of shear deformations of the order of $10^{-4}-10^{-2} \%$, and also allow you to identify changes in these indicators in the range of small shear deformations.

3. The obtained results allow to perform dynamic calculations of the bases of buildings and structures, composed of coarse-grained soils, in seismically dangerous territories. At present, such calculations are performed mainly by numerical calculations using the FEM, implemented by special geotechnical software systems.

This work was financially supported by Ministry of Education and Science of the Russian Federation (1.4984.2017/6.7). All tests were carried out using research equipment of The Head Regional Shared Research Facilities of the Moscow State University of Civil Engineering (RFMEFI59317X0006).

\section{References}

1. T. Wichtmann Explicit accumulation model for non-cohesive soils under cyclic loading. (Bochum, 2005)

2. E. Voznesenskij Dynamic testing of soils. Situation and standardization. J. Engineering survey 5, p. 20-26 (2013)

3. J. Yamamuro, P. Lade Static liquefaction of very loose sands. Can. Geotech. J. 34, 6. p. $905-917$ (1997)

4. K. Ishihara The behavior of soils during earthquakes (St. Petersburg, NGO «Georeconstruction-Fundamentproject», 2006)

5. E. Sobolev, A. Ter-Martirosjan Effect of physical properties of sandy soil on the dynamic stability of the bases of buildings and structures. XIX International interuniversity scientific-practical conference of students, undergraduates, graduate students and young scientists «Building - the formation of living environment». National Research Moscow State University of Civil Enginering. Moscow p. 10871090 (2016)

6. A. Ter-Martirsjan, A. Mirnyj, E. Sobolev Features of determining the parameters of modern soil models in laboratory tests. Geotechnics J. 1. p. 66-72 (2016) 
7. J. A. Schneider, P. W. Mayne Soil liquefaction response in mid-America evaluated by seismic piezocone tests, Technical report, Mid-America Earthquake Center Report MAE-GT-3A Atlanta (1999)

8. F. Y. Menq Dynamic Properties of Sandy and Gravelly Soils (2003)

9. M. Goudarzy Micro and Macro Mechanical Assessment of Small and Intermediate Strain Properties of Granular Material (2015)

10. K. Iida The velocity of elastic wave in sands. Earthquake res. ins. J. p. 132-145 (1937)

11. B. O. Hardin, J. G. Music Apparatus for testing effects of torsional vibration (1965)

12. F. Jafarzadeh and H. Sadeghi Experimental study on dynamic properties of sand with emphasis on the degree of saturation. Soil Dyn. and Earthquake Eng. 32. p. 26-41. (2012)

13. J. Camacho-Tauta, Evaluation of the small-strain stiffness of soil by nonconventional dynamic testing methods, PhD Thesis, Technical University of Lisbon (Lisbon, 2011)

14. M. Goudarzy, D. König, C. Santamarina, T. Schanz Influence of anisotropic stress state on intermediate strain properties of granular material. Géotechnique J. 6. p. 180 (2017)

15. Y.-S. Bae, J. A. Bay, Modifications of Resonant Column and Torsional Shear Device for the Large Shear Strain. Computers and Geotechnics J. 36. p. $944-952$ (2009)

16. Z. Khan, G. Cascante, J. Hutchinson, P. Pezeshkpour Evaluation of the First Mode of Vibration and Base Fixidity in Resonant-Column Testing. ASTM Geotech. Test. J. 31(1). (2008)

17. Z. H. Khan, G. Cascante, M. H. El Naggar, C. G. Lai Measurement of FrequencyDependent Dynamic Properties of Soils Using the Resonant-Column Device. J. of Geotech. and Geoenv. Eng., ASCE. 134(9). p. 1319-1326 (2008)

18. T. Qiu, P.J. Fox Effective Soil Density for Propagation of Small Strain Shear Waves in Saturated Soil. J. of Geo. and Geoenv. Eng., ASCE. 134(12). p. 1815-1819 (2008)

19. A. Viana da Fonseca, C. Ferreira, M. Fahey A Framework Interpreting Bender Element Tests, Combining Time-Domain and Frequency-Domain Methods. Geo. Test. J.. ASTM, 32(2). p. 1-17. (2009)

20. S. Yamashita, T. Kawaguchi, Y. Nakata, T. Mikami, T. Fujiwara, S. Shibuya Interpretation of International Parallel Test on Measurement of Gmax Using Bender Elemens. Soils and Found. J. 49(4). p. 631-650 (2009)

21. ASTM International. Annual Book of ASTM Standards (West Conshohocken, 2002) 